Annuaire du Collège de France 2017-2018

\title{
Christian Goudineau (1939-2018)
}

Jean-Pierre Brun

\section{(2) OpenEdition}

Journals

Édition électronique

URL : https://journals.openedition.org/annuaire-cdf/16625

DOI : 10.4000/annuaire-cdf. 16625

ISBN : 978-2-7226-0572-5

ISSN : 2109-9227

Éditeur

Collège de France

Édition imprimée

Date de publication : 30 décembre 2020

Pagination : 735-736

ISBN : 978-2-7226-0516-9

ISSN : 0069-5580

Référence électronique

Jean-Pierre Brun, «Christian Goudineau (1939-2018) », L'annuaire du Collège de France [En ligne], 118 2020, mis en ligne le 01 avril 2021, consulté le 22 août 2022. URL : http://journals.openedition.org/ annuaire-cdf/16625 ; DOI : https://doi.org/10.4000/annuaire-cdf.16625 


\section{CHRISTIAN GOUDINEAU (1939-2018)}

Notre collègue Christian Goudineau est décédé le 9 mai 2018. Il fut un personnage central de l'archéologie française pendant quarante ans. Il m'est impossible de résumer en quelques minutes son parcours et son œuvre scientifique, littéraire et politique - au sens de politique de la recherche. Je vais donc dégager quelques traits saillants, vous renvoyant à l'article de fond que Jean Guilaine, Michel Gras, Vincent Guichard et moi-même préparons pour la revue Gallia.

Christian Goudineau naquit en 1939. Il fit de brillantes études supérieures aboutissant à l'agrégation de lettres classiques en 1962. De 1965 à 1968, il fut membre de l'École française de Rome, qui est l'institut de formation supérieur des historiens et archéologues. Là, il écrivit une thèse remarquée sur l'évolution d'une catégorie essentielle de céramique romaine. En 1968, il fut nommé à l'université de Provence, puis passa son doctorat d'État en 1975 sur le processus de romanisation à partir de ses recherches archéologiques à Vaison-la-Romaine dans le Vaucluse. Il devint alors professeur d'archéologie nationale à Aix-en-Provence et écrivit plusieurs ouvrages dont une brillante synthèse sur l'histoire urbaine en Gaule romaine qui le fit remarquer. Enfin, de 1984 à 2010, il fut professeur au Collège de France, titulaire de la chaire Antiquités nationales, c'est-à-dire consacrée à l'archéologie de la France. De ce poste, il rayonna durant un quart de siècle sur l'ensemble de la discipline en France et en Europe.

L'homme avait de nombreuses facettes. Vous me voyez obligé de m'en tenir aux têtes de chapitres et à quelques rappels.

Le chercheur. Il était un archéologue très attentif aux contextes historiques et matériels. Il s'était spécialisé dans la protohistoire récente de la Gaule et dans les débuts de la présence de Rome, fouillant à Vaison, à Taradeau et le camp de la flotte romaine à Fréjus. Il publia plusieurs centaines d'articles scientifiques et une quinzaine d'ouvrages parmi lesquels un César et la Gaule et Le dossier Vercingétorix qui font autorité.

L'enseignant était hors du commun par sa capacité de synthèse des travaux les plus récents, éclairés par sa profonde connaissance de la littérature classique et par une structuration mentale hors pair. Il brillait non seulement dans ses cours magistraux mais aussi dans les séminaires qu'il organisait à l'université d'Aix avec Paul-Albert Février, attirant des chercheurs de toute la moitié sud de la France. Au Collège de France, il élargit encore son audience. Il illuminait donc, mais pas seulement : il savait écouter les étudiants, les encourager, tirer le meilleur d'eux-mêmes, décelant ceux, même parmi les plus humbles, qui avait le potentiel pour devenir de vrais 
archéologues, c'est-à-dire des historiens maniant les archives du sol. Il les conduisait du terrain à la reconnaissance universitaire en dirigeant leur thèse.

L'administrateur de la recherche. Dès son arrivée à Aix, il saisit l'opportunité de devenir, en plus de professeur, directeur des Antiquités de la Côte d'Azur pour le compte du ministère de la Culture. C'est une responsabilité qui lui permit de développer ses propres fouilles, mais surtout d'impulser la recherche dans le sens d'une plus grande préservation du patrimoine au service d'une meilleure connaissance de notre passé. À ce poste, il encouragea et forma de nombreux jeunes chercheurs et eut à négocier avec des hommes politiques et des aménageurs, réussissant souvent à faire respecter la loi malgré les puissances d'argent. En exerçant ces responsabilités au contact de la réalité économique et politique, il put mesurer les insuffisances de la réglementation et la nécessité de la changer.

Cela ne suffisant pas, il fut aussi directeur de l'UER «Lettres » de l'université de Provence de 1975 à 1979, puis directeur, avec Jean Guilaine, du laboratoire du CNRS en charge de la publication de la revue Gallia (1984-1994).

Le "politique de la recherche ». Dès avant sa nomination au Collège de France, fort de son expérience de directeur des Antiquités, il combattait pour imposer des fouilles préventives avant tout aménagement destructeur. En 1985, il fut nommé vice-président du Conseil supérieur de la Recherche archéologique présidé par Jack Lang, et parallèlement, de 1985 à 2001, il présida le Conseil scientifique du centre européen du Mont-Beuvray. En 1989-1990, il profita de « l'année de l'archéologie », pour promouvoir les récents acquis de l'archéologie française dans un grand ouvrage De Lascaux au Grand-Louvre (Errance, 1991) codirigé avec Jean Guilaine. En 1990, à la demande de Michel Rocard, alors Premier Ministre, il écrivit un rapport décisif sur l'archéologie préventive et il fut l'un des acteurs du renforcement de la législation aboutissant à la convention de Malte en 1992 et à la loi sur l'archéologie préventive en 2001.

Le romancier. Christian Goudineau était un pédagogue. Toute sa vie, il s'est attaché à diffuser la connaissance historique dans le grand public par des conférences, des diaporamas, des reportages, des ouvrages de vulgarisation. À partir de 2000, il s'est mué en romancier pour accroître son audience au-delà du monde universitaire. Un premier roman, Le Voyage de Marcus : les tribulations d'un jeune garçon en Gaule romaine (Actes Sud, 2005) emmène le lecteur à travers la Gaule romaine. D'autres ont suivi tels que L'Enquête de Lucius Valérius Priscus (Actes Sud/ Errance, 2004 ; Babel 2007) et Le Procès de Valérius Asiaticus (Actes Sud, 2011) qui font découvrir les mœurs romaines.

L'homme enfin était un personnage solaire et joyeux. Il aimait les fêtes et en donnait souvent. Il y brillait par sa conversation, bien sûr, mais aussi par les chansons qu'il était capable d'écrire à chaud, notamment lors de congrès, chansons qu'il chantait excellemment lors des banquets finaux. Plus profondément, il était très attentif à la vie, à la carrière de ses étudiants et collaborateurs, mettant tout en œuvre pour assurer leur succès universitaire, repérant les talents, les poussant à se dépasser, les aidant de toutes les manières possibles aussi bien intellectuellement que financièrement. C'était un grand maître dans tous les domaines.

Je terminerai par une citation de Christian Goudineau lui-même : « Ma conviction, c'est que l'historien et l'archéologue ont pour vocation de proposer des cadres structurants à leurs concitoyens et aux enfants. » C'est à cette tâche qu'il s'est voué toute sa vie. 